\title{
Aging and Periodontium
}

\section{Avnika Garg ${ }^{1}$ \\ Shivani Bhickta ${ }^{2}$}

\section{Rajesh Kumar Gupta ${ }^{3}$ \\ Alka Sharma ${ }^{4}$}

\section{ABSTRACT}

Diseases of the periodontium occurs in the childhood, adolescence and early adulthood, but the prevalence of periodontal diseases and tissue destruction increases with age. The increase may be caused by the cumulative effect of the number of bursts of periodontal destruction, deterioration in plaque removal efficiency, or an increase in the number of teeth retained in old age and therefore affected by plaque-induced disease. But with appropriate periodontal therapy and self-performed plaque control, older patients can be expected to have as favorable treatment outcomes as young patients.

\section{INTRODUCTION}

The periodontium consists of the investing and supporting tissues of the tooth (gingiva, periodontal ligament, cementum, and alveolar bone). The periodontium is subject to morphologic and functional variations as well as changes associated with age. ${ }^{4}$ Many tissue changes occur with aging, some of which may affect the disease experience of the periodontium. ${ }^{2}$ Diseases of the periodontium occurs in the childhood, adolescence and early adulthood, but the prevalence of periodontal diseases and tissue destruction increases with age. Aging is a slowing down of the natural function, a disintegration of the balanced control and organization that characterize the young. ${ }^{49}$ It is a process of physiologic and morphologic disintegration, as distinguished from infancy, childhood, and adolescence, which are typified by processes of integration and coordination. ${ }^{2}$ Senescence is a term used to include changes in the adult organism that occur with time. ${ }^{20}$

\section{AGING AND ITS EFFECTS ON WHOLE BODY}

Aging of an organ may be defined as the post maturational deteriorative changes that with time leads to an increased vulnerability to challenges, thereby decreasing the functional ability of the organ. ${ }^{16}$ Aging is manifested to different degrees and in different manners in various tissues and organs, but it includes general features such as: tissue dessiccation, reduced elasticity, diminished reparative capacity, altered cell permeability and increased calcium content in the cells of many organs. ${ }^{2}$ Arteriosclerosis is a frequent finding in aging humans, and may be seen in large vessels with muscular elements in the vessel wall, vessels in the alveolar bone, and vessels in the periodontal ligament. The relationship of this vascular pathologic condition to other changes in the periodontium is inconclusive. The relative ischemia that arteriosclerosis may produce in periodontal tissues because of the reduction in blood flow has been hypothesized as predisposing these tissues to disease or provoking other changes such as fibrosis, loss of cellularity, and focal calcification. It may also reduce bone metabolism and may be correlated with slower or altered wound healing. ${ }^{34}$

\section{AGING AND ORAL MUCOSA}

The keratinization potential of the hard palate epithelium does not change with age. An increased keratinization of lip and cheek mucosa with age has been reported. This might, however, be related to smoking. ${ }^{2}$ Changes in other areas of the oral mucosa include atrophy of the epithelium and connective tissue with loss of elasticity, decrease in proteinbound hexoses and mucoproteins, which may reduce resilience and increase susceptibility to trauma; increase in mast cells. ${ }^{1}$ Elastoid degeneration is reported in the collagen fibers of the alveolar mucosa. ${ }^{20}$ Atrophy of the papillae of the tongue, with the filiform papillae more severely affected; decrease in the number of taste buds in the circumvallate papillae, nodular varicose enlargement of veins on the ventral surface of the tongue; and increase in the sebaceous glands in the lip and cheek. Regressive changes in the salivary glands with retention cyst formation and associated xerostomia have been identified with aging. ${ }^{1}$

\section{AGING AND GINGIVA}

As the age increases, there is an increased prevalence of gingival recession. But this finding appears to be largely due to the cumulative environmental effects of vigorous tooth brushing rather than due to the inflammatory periodontal disease. With continuing gingival recession, the width of the attached gingiva would be expected to decrease with age, but the opposite appears to be true. ${ }^{5}$ As the age increases, thinning of gingival epithelium has been reported to occur. ${ }^{49}$ Diminished keratinization of gingival epithelium has been reported in both men and women. ${ }^{2}$ In menopausal patients the gingiva is less keratinized than in patients of comparable age with active menstrual cycles. ' Conflicting results have been published regarding the shape of retepegs. 
Shklar(1966) reported a flattening of rete-pegs, where as Wentz et al(1952) found that the height of the epithelial ridges increased with age. Wentz et al (1952) found that the irregular, finely textured pre-pubertal connective tissue shows a gradual transition with age to a denser, coarsely textured tissue in the higher age group connective tissue. ${ }^{49}$ Decreased connective tissue cellularity; a greater amount of intercellular substances and reduced oxygen consumption is a measure of reduced metabolic activity. There is reduced or unchanged amount of stippling. ${ }^{2}$ Rate of collagen synthesis is decreased, whereas, rate of maturation of the synthesized collagen is increased with age. So, there is an increase in the thickness of collagen fibrils, thus leading to increased tensile and mechanical strength. Some of these changes may be related to the loss of acid mucopolysaccharide and water as well as to increased cross-linkages. ${ }^{20}$

\section{AGING AND PERIODONTAL LIGAMENT}

With increasing age, the fiber and cellular contents decrease and the structure of the ligament becomes more and more irregular. ${ }^{49}$ Principal fibers get thickened and bundles become wavy and broad. Young collagen takes up silver nitrate readily (argyrophilia) whereas old collagen takes up the stain only slightly. ${ }^{20}$ There is decreased number of cell density and reduction in organic matrix production with advancing age. ${ }^{49}$ PDL shows degenerative hyaline changes and at times, cells within lacunae are demonstrable. Calcified bodies become common and epithelial rests show altered forms of aggregation. ${ }^{20}$ Conflicting results have been reported for changes in the width of periodontal ligament in relation to age in human and animal models. Although true variation might exist, this finding probably reflects the functional status of the teeth in the studies because the width of the space will decrease if the tooth is unopposed (hypofunction) or will increase with excessive occlusal loading. Moreover, Ive et al. (1980) also noted that both the width of the socket and the width of the cementum increased with age. They suggested that, as the socket remodels and increases in size with age, cementum is deposited at a relatively greater rate than the bone, causing a decrease in the periodontal ligament space. ${ }^{49}$

\section{AGING AND CEMENTUM}

With age, the cementum increases in width. The total width of cementum at age 76 is three times that at age $11 .^{2}$ This finding is not surprising because cementum deposition continues after tooth eruption and occurs throughout the life of man. The increase in width is greater apically and lingually. ${ }^{5}$ Indications exist that cemental deposition slows in old age..$^{20}$ An older root surface is less highly populated with fiber bundles than a younger root surface. ${ }^{31}$ Local resorption at the cementum surface followed by cementum apposition is often observed leading to increase in surface irregularity. Compared with other cells of the periodontal tissues, cementocytes normally exhibit the lowest proliferative capacity. ${ }^{49}$ Seen microscopically, only the surface layer of cementocytes appears viable. All other lacunae appear empty. ${ }^{31}$ Spurring of cementum is sometimes the result of the fusion of calcospheroid near cementum or of the calcification of epithelial rest aggregates. ${ }^{20}$ With increasing age, the process of cementum formation becomes essentially acellular. ${ }^{49}$ Attachment of cementum to dentin may be weakened with increasing age. Frequent cemental tears seen in specimens of aging humans may be related to the age changes in the ground substance of cementum, to reduced vascularity, or to thickened and less extensible ligament fibers embedded in the cementum. ${ }^{20}$

\section{AGING AND ALVEOLAR BONE}

Little evidence of continued bone apposition is present in senescence. In view of this, physiologic tooth migration may be slowed or even halted in old age. ${ }^{20}$ Reduction in bone height occurs with aging (senile atrophy). It has been noted that, with increasing age, the periodontal surfaces of the alveolar bone become jagged and that collagen fibers insert less regularly in bone. ${ }^{49}$ Bone undergoes osteoporosis with aging. Although age is a risk factor for the bone mass reductions in osteoporosis, it is not causative and therefore should be distinguished from physiologic aging processes. Other changes in alveolar bone include: bone is rarefied (Loss of bone mineral density), trabeculae are reduced in numbers, cortical plates are thinned, vascularity is decreased, water content of bone is reduced, collagen fibers are thickened and bone formation is decreased. Hence, there is reduction in metabolism and so increased susceptibility to fractures. ${ }^{49}$

\section{AGING AND BACTERIAL PLAQUE}

Dentogingival plaque accumulation has been suggested to increase with age. This might be explained by the increase in hard tissue surface area as a result of gingival recession and the surface characteristics of the exposed root surface as a substrate for plaque formation compared with enamel. For supra-gingival plaque, no real qualitative differences have been shown for plaque composition. For subgingival plaque, one study has shown similar subgingival flora to a normal flora, whereas another study reported increased numbers of enteric rods and pseudomonas in older adults. It has been speculated that a shift occurs in the importance of certain periodontal pathogens with age, specifically including an increased role for Porphyromonas gingivalis and a decreased role for Actinobacillus actinomycetemcomitans. However, 
differentiating true age effects from the changes in ecological determinants for periodontal bacteria will be difficult. ${ }^{5}$

\section{EFFECTS OF AGING ON PROGRESSION OF PERIODONTAL DISEASE}

The phrase "getting long in the tooth" expresses a widespread belief that age is inevitably associated with an increased loss of connective tissue attachment. However, this observation might equally well reflect a cumulative exposure to a number of potentially destructive processes. These exposures might include plaque associated periodontitis, chronic mechanical trauma from tooth brushing, and iatrogenic damage from unfavorable restorative dentistry or repeated scaling and root planing. The effects of these exposures act in one direction only (i.e. increased loss of attachment). In this experimental model, a comparison of developing gingivitis between young and older individuals demonstrated a greater inflammatory response in older subjects, both in humans and dogs. In the older age group ( 65 to 80 years), the findings included a greater size of infiltrated connective tissue, increased gingival crevicular fluid flow, and increased gingival index. ${ }^{6}$ Although the amount of periodontal tissue destruction, measured as clinical attachment loss may be greater in an 80 year-old individual than in an 18-year-old, the young patient may suffer from more severe disease and may lose more attachment in a short period. If left untreated, such an individual will lose the most heavily affected teeth early in life, whereas the more resistant ones will be maintained for a longer period. ${ }^{42}$

\section{AGING AND IMMUNE RESPONSE}

Differences between young and older individuals can be demonstrated for $\mathrm{T}$ and $\mathrm{B}$ cells, cytokines, and natural killer cells but not for polymorphonuclear cells and macrophage activity. McArthur concludes, "Measurement of indicators of immune and inflammatory competency suggested that, within the parameters tested, there was no evidence for agerelated changes in host defenses correlating with periodontitis in an elderly (65 to 75 years) group of individuals, with and without disease." In summary, although many contradictions exist, a survey of the literature demonstrates that some age related changes are evident in the periodontium and host response. In other words, the rate with which the inflammation of the periodontium develops, increases with age. ${ }^{5}$

\section{AGING AND WOUND HEALING}

Rate of healing of wounds decreased with increasing age probably connected to a slower restitution of the microvascular system. The effect of aging was most pronounced for the connective tissue regeneration. The gingival tissues never regenerated completely in the older age group. In response to treatment of periodontitis, older patients generally have all the needed cellular and molecular mechanisms to ensure healing and repair, but these mechanisms may be slower and somewhat lower than those in young patients. ${ }^{5}$

\section{AGING AND THE CUMULATIVE EFFECTS OF ORAL DISEASE}

With time chronic diseases can produce many oral changes, and it is difficult to determine how much physiologic aging contributes to the total picture. Some believe that gingival recession, attrition and reduction in bone height in the aged results more from disease and factors in the oral environment than from physiologic aging. Although recession, attrition and bone loss commonly occur with the age, they are not present in all patients, and vary considerably in the same age group. An aged individual with marked attrition may present relatively little bone loss. Marked attrition may also be produced in young and middle aged adults by bruxing and clenching habits. Increased alveolar bone loss in the aged has been related to less efficient oral hygiene. Bone loss, pathologic migration of the teeth, and loss of the vertical dimension in the aged may be the results of periodontal disease and failure to replace missing teeth. ${ }^{2}$ Leukoplakia of the oral mucosa and staining of the teeth are common in aged individuals who are inveterate smokers. Wearing artificial dentures for years without rebasing, with a resultant reduction in vertical dimension, is a common cause of angular cheilosis in the aged. ${ }^{1}$

\section{AGING AND RESPONSE TO THE TREATMENT OF THE PERIODONTIUM}

The studies that have been done in relation with this aspect of aging clearly demonstrate that despite the histologic changes in the periodontium with aging, no differences in response to nonsurgical or surgical treatment have been shown for periodontitis. However, if plaque control is not ideal, continued loss of attachment is inevitable. Aging might affect other aspects of managing the periodontal diseases, such as the risk of root caries, and the resulting difficulties should not be underestimated. Interestingly, a recent study has identified greater compliance with supportive maintenance among older individuals than younger patients. $^{2}$

\section{AGING AND PROGNOSIS}

It is generally believed that in patients with the same amount of periodontal disease, the rule holds: the older the patient, the better the prognosis in terms of no recurrence of the disease (Goldman \& Cohen 1973, Schluger et al, 1977). This 
belief is based mainly on the reasoning that the resistance to periodontal breakdown must be higher in older individuals, since the process of periodontal destruction takes much less time in younger individuals. ${ }^{49}$

\section{CONCLUSION}

Periodontal tissues are programmed to provide healthy support for the dentition for the life of the patient. Periodontal disease increases in prevalence and severity with increasing age. The increase may be caused by the cumulative effect of the number of bursts of periodontal destruction, deterioration in plaque removal efficiency, or an increase in the number of teeth retained in old age and therefore affected by plaque-induced disease. Although there are concerns that older patients have increased susceptibility to periodontal disease, role of age in disease progression is minimal. With appropriate dental therapy, and maintenance of low plaque levels, clinicians can assure older patients that they can keep a functioning dentition even though there may be a slightly lower resistance to bacterial induced periodontal inflammation and higher levels of putative perio-pathogens present.

\section{ACKNOWLEDGMENT}

We thank Dr. Nandini Nayyar, Senior Lecturer, Dept. of Periodontics, Bhojia Dental College and Hospital for reviewing the manuscript and helpful discussion.

\section{REFERENCES}

1. Glickman's Clinical Periodontology, 5th edition by Fermin A. Carranza and Jr. Dr. Odont

2. Glickman's Clinical Periodontology, 7th edition by Fermin A. Carranza and Jr. Dr. Odont

3. Clinical Periodontology, 8th edition by Newman and Carranza.

4. Carranza's Clinical Periodontology, 9th edition by Newman, Takei, Klokkevold and Carranza.

5. Carranza's Clinical Periodontology, 10th edition by Newman, Takei, Klokkevold and Carranza.

6. Carranza's Clinical Periodontology, 11 th edition by Newman, Takei, Klokkevold and Carranza.

7. Essentials of Periodontology and Periodontics, 2nd edition, by Shantipriya Reddy

8. Essentials of Periodontology and Periodontics, 3rd edition, by Shantipriya Reddy

9. Clinical Periodontolgy and Implant Dentistry, 5th edition, by Jan Lindhe

10. Clinical Periodontolgy and Implant Dentistry, 4th edition, by Jan Lindhe

11. Periodontal Therapy, 6th edition, By Henry M. Goldman, D. Walter Cohen

12. The Periodontic Syllabus, 5th edition, by R.Vernino, Jonathan Gray, Elizabeth Hughes

13. Periodontics, 6th edition, by B.M.Eley, M.Soory, J.D.Manson

14. Periodontics, 5 th edition, by B.M.Eley and J.D.Manson

15. Outline of Periodontics, 4th edition, by J.D.Manson and B.M.Eley

16. Fundamentals of Periodontics by Thomas G. Wilson and Kenneth S. Kornman
17. Critical Decisions in Periodontology, 4 th edition, by Hall

18. Contemporary Periodontics, by Robert J. Genco, Henry M. Goldman, D. Walter Cohen.

19. Periodontology and Periodontics: Modern Theory and Practice, 1st edition, by Sigurd P. Ramfjord and Major M. Ash.

20. Periodontics, 5th edition, by Daniel A. Grant, Irving B. Stern, Frank G. Everett

21. Periodontics, A Synopsis, by W M M Jenkins and C J Allan

22. Current Concepts in periodontics, 1st edition, by B.R.R. Varma, R.P.Nayak

23. Textbook of Periodontology, 2nd edition, by Gruraja Rao

24. Manual of Clinical Periodontics, Howard L. Ward and Marvin Simring

25. Color Atlas of Dental Medicine, Periodontology, 2nd edition, by Klaus H. \& Edith M. Rateitschak, Herbert F. Wolf, Thomas M. Hassell

26. Drugs, diseases and the Periodontium, by Robin A. Seymour, Peter A. Heasman, Ian D.M. Macgregor

27. Clinical Periodontology for Dental Hygienist, by Perry and Carranza.

28. Foundations of Periodontics for the Dental Hygienist, 3rd edition, by Jill S. Nield Gehrig, Donald E. William

29. Orban's Oral Histology and Embryology, 13th edition, by G.S.Kumar

30. Oral Anatomy, Histology and Embryology, 4th edition, by B.K.B.Berkovitz, G.R.Holland and B.J.Moxham

31. Oral Development and Histology, 3rd edition, by James K.Avery

32. Tencate's Oral histology, 7th edition, by Antonio Nanci

33. Oral Histology-Inheritance and Development, 2nd edition, by D. Vincent Prowenza and Werner Seibel

34. Textbook of Periodontology and Oral Implantology, by Dilip G Nayak, Ashita Uppoor and Mahesh C.P.

35. Color Atlas and Text of Dental care of the Elderly by John R Drummond, James P Newton and Robert Yemm

36. Periodontal and Gingival Health and Diseases children, adolescents and young adults, by Enrique Bimstein, Howard L Needleman, Nadeem Karimbux and Thomas E Van Dyke.

37. A Guide to Elderly Care by Dr. O.P. Sharma

38. Edward J. Ryan, Patrick D. Toto, and Anthony W. Gargiulo. Aging in human attached gingival epithelium. JDR 1972; 53:1: 74-76.

39. Alveolar bone loss and Aging: A model for the study in mice. J Periodontol 2005; 76: 1966-1971.

40. Casual factors in shortening tooth series with age. JDR; 17:1:1-13.

41. The effect of age on proliferating cell nuclear antigen expression in oral gingival epithelium of healthy and inflamed human gingiva. $J$ Periodontol 2000; 71: 1567-1574.

42. Aging and the periodontal and peri-implant microbiota. Perio 2000; 16: 44-52.

43. Treatment of periodontal disease in older adults. Perio 2000; 16 : 106-112.

44. Thickness of palatal masticatory mucosa associated with age. J Periodontol 2001; 72: 1407-1412

45. Effect of aging on immunocompetent and inflammatory cells. Perio $2000 ; 16: 53-79$.

46. The potential for increase in the periodontal diseases of the aged population, J Periodontol 1983; 54:12:721-730.

47. Frank M.Wentz, Alexis W.Maler and Balint Orban. Age changes and Sex differences in the clinically healthy ginigva. JOP: 13-24.

48. Effect of aging on functional changes of periodontal tissue cells. Ann Periodontol 1998; 3: 350-369.

49. Effect of age on periodontium. JCP 1984; 11: 281-294. 